\title{
EXPERIMENTAL STUDY OF NEOCLASSICAL PLASMA FLOW AND BOOTSTRAP CURRENT IN THE TOKAMAK TEXTOR
}

\author{
K. HÖTHKER, H.-J. BELITZ, R.P. SCHORN \\ Institut für Plasmaphysik, Forschungszentrum Jülich $\mathrm{GmbH}$, \\ Association Euratom/KFA, \\ Jülich, Germany \\ W. BIEGER \\ Fachhochschule Aachen, Abt. Jülich, \\ Jülich, Germany \\ J.A. BOEDO \\ Institute of Plasma and Fusion Research, \\ University of California at Los Angeles, \\ Los Angeles, California \\ United States of America
}

\begin{abstract}
An experimental study of ion flow in the edge plasma of the tokamak TEXTOR has been performed. The corresponding predictions of neoclassical theory could be verified qualitatively to a large extent and quantitatively for small densities. The measurements indicate that the observed drift of the ions is due to the neoclassical toroidal plasma flow and ion bootstrap current. The energy density of the drift is significant compared with the total thermal energy density. Application of the model developed for the edge plasma allows an extensive understanding of the characteristic features of the particle exhaust by the pump limiters on TEXTOR.
\end{abstract}

\section{INTRODUCTION}

The study of plasma rotation in tokamaks is of interest as a check for the validity of neoclassical theory in general and for the understanding of the particle and energy transport in particular, for example, Hinton and Hazeltine [1] and Balescu [2]. For example, plasma rotation seems to have a decisive importance for the establishment of an $\mathrm{H}$ mode, Burrell et al. [3]. The main subject of this paper is an experimental study of the toroidal ion flow in the edge plasma for ohmic discharges in the tokamak TEXTOR and a comparison with tokamak theory. Neoclassical theory predicts for the present plasma conditions a toroidal rotation which is a superposition of a plasma flow, due to angular momentum balance, and an ion current, due to the bootstrap effect. The dependence of the first contribution on the collisionality of the ions is much weaker than that of the second contribution. Although the origin of the two contributions is quite different their dependences on the driving forces, such as the radial gradients of density and temperature, are similar. This fact makes a quantitative investigation of the two contributions difficult, the more so as theoretical results for the bootstrap current have not yet been elabo- rated for the finite aspect ratio and collisionality regime in the plasma of the present study. The existence of bootstrap currents in tokamaks has been deduced up to now from code interpretations of measurements, for example, for TFTR Zarnstorff et al. [4] and for JET Kim et al. [5]. A measurement of the speed of toroidal plasma rotation - integrated over the plasma cross-section - has been published for one particular ohmic discharge by Bell [6] and by Bugarya et al. [7]. Both results are reported to be consistent with the neoclassical predictions.

Usually the plasma flow is determined indirectly. Sometimes it is determined from the measured radial electric field assuming that the $E \times B$ term is the leading one in the angular momentum balance [7]. This assumption is not correct in general, Hazeltine [8], and especially not for the present investigation. Sometimes the plasma flow velocity is inferred from the measurement of the Doppler shift of impurity lines which may be, however, significantly misleading, Kim et al. [9].

In this paper we present an experimental study of the Mach number in the edge plasma of the tokamak TEXTOR for ohmic discharges by means of a rotating electrical double probe, acting as a Mach probe. 


\section{HÖTHKER et al.}

\section{EXPERIMENTAL SET-UP AND DIAGNOSTIC METHODS}

We have developed the rotating electrical double probe to perform calibrated density measurements in magnetized plasmas [10] and to perform ion temperature measurements in non-streaming magnetized plasmas $[11,12]$. For streaming, magnetized plasmas the probe can be applied to perform Mach number measurements [13]. Figure 1 shows a cross-section of the probe head. The two probe pins are identical cylinders with a length of $5 \mathrm{~mm}$, a diameter of $5 \mathrm{~mm}$ and a distance between their axes of $10 \mathrm{~mm}$. They are made of graphite. The probe rotates during a discharge with a frequency of $2 \mathrm{~Hz}$ about its axis which is perpendicular to the magnetic field of the tokamak. It is mounted in the equatorial plane of the tokamak TEXTOR (Fig. 2) and can be moved in the

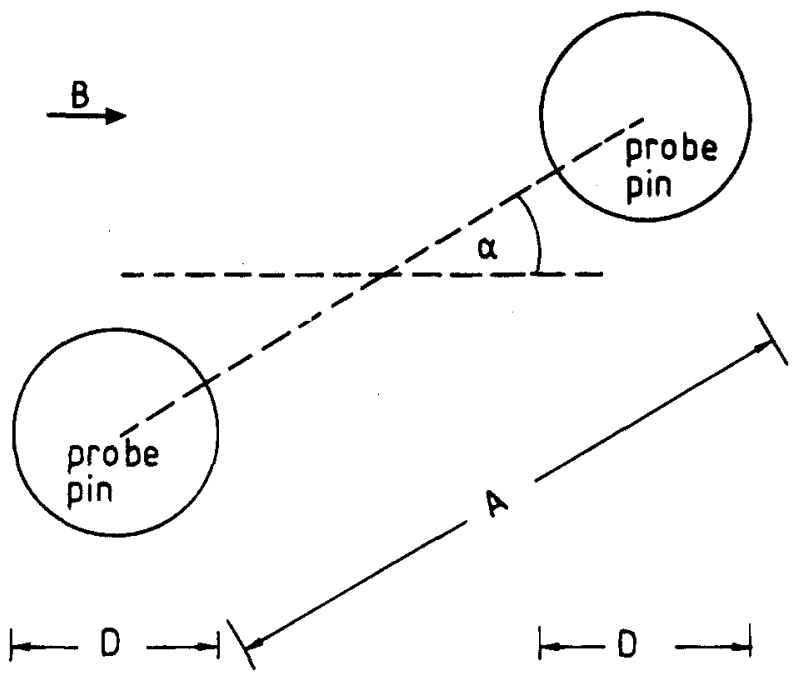

FIG. 1. Configuration of the pins of the rotating probe in a magnetic field.

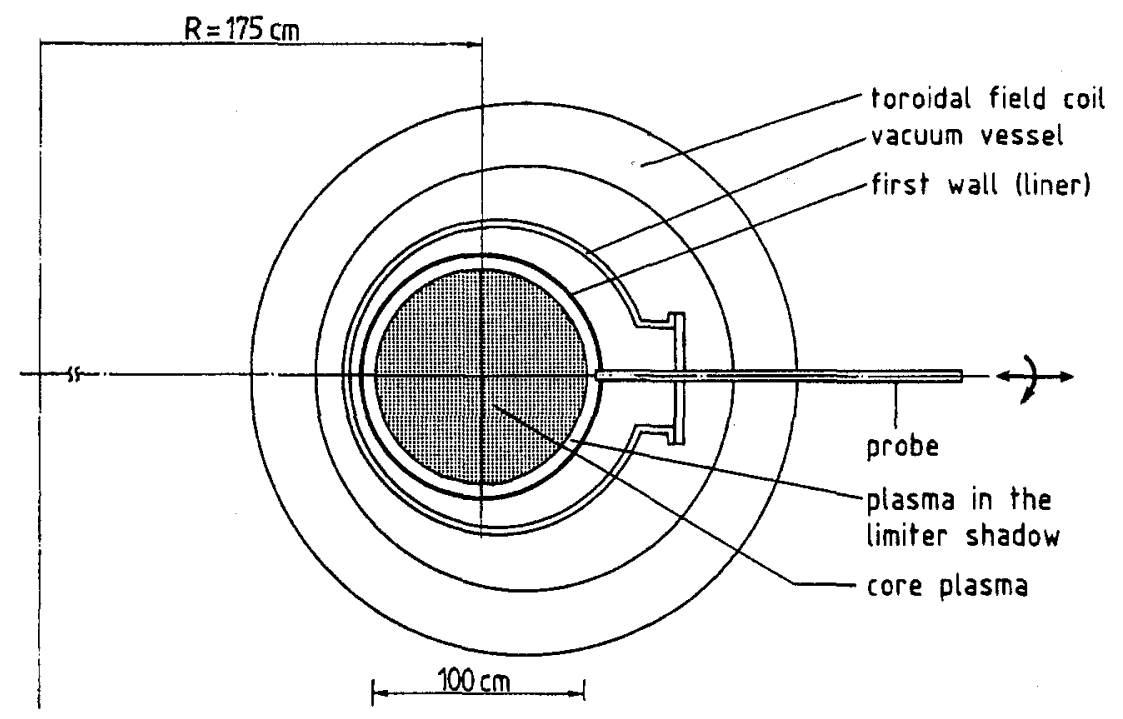

FIG. 2. Position of the rotating probe on the tokamak TEXTOR.

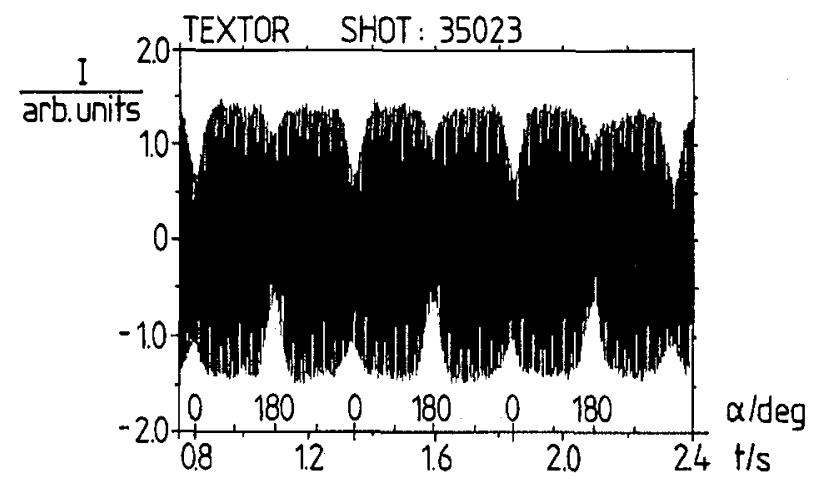

FIG. 3. Ion saturation current of the probe $I_{s a t}$ as a function of the angle $\alpha$. radial direction. The probe signals are generated in the conventional way in applying a triangular voltage with a frequency of $200 \mathrm{~Hz}$ to the probe pins generating an electrical current from which electron temperature and density can be deduced as usual. Figure 3 shows the envelope of the current signal, i.e. the ion saturation current as a function of time and the angle $\alpha$ (Fig. 1). Each time when one probe pin lies in the shadow of the other, i.e. $\alpha=0$ and $\pm 180^{\circ}$, the ion saturation current has a minimum value. The up/down asymmetry of the depression is interpreted as due to an ion flow as for conventional Mach probes: the probe pin towards the upstream side collects more ions than the pin on the 
downstream side, so that the direction of the current can be determined. A measurement of the ratio of the ion saturation currents on the upstream and downstream sides allows a determination of the Mach number. To this end we use the theoretical results of Chung and Hutchinson [14]. This theory assumes Maxwellian velocity distributions for the ions. In this respect we rely on a result of Hinton and Hazeltine [1] and Balescu [2] that even in the long mean free path regime the velocity distribution of the particles is Maxwellian, in leading order of the drift parameter which is in fact small $(\ll 1)$ for the present conditions.

A further precondition of Ref. [14] is that the mean gyroradius of the ions is small compared with the probe pin radius. From our former measurements [15] one cannot expect that this condition is strictly fulfilled. This fact implies that the Mach numbers as determined in this paper are lower limit values.

The edge of the plasma is defined by limiters. For the measurements presented in this paper a poloidal limiter which has an extension of $35^{\circ}$ in the poloidal direction is located at $r=44 \mathrm{~cm}$. The position of the probe is virtually in the plane of symmetry between the two sides of the poloidal limiter. The distance to the two sides along the magnetic field lines is about 60 and $80 \mathrm{~m}$, respectively. For $r>46.7 \mathrm{~cm}$ the probe measures in the shadow of the toroidal ALT-II limiter. (The quoted radii take into account the shift due to the different temperatures of the TEXTOR vessel.) ALT-II consists of 8 blades mounted $45^{\circ}$ below the equatorial plane. The extension of each blade is $1.50 \mathrm{~m}$ in the toroidal direction, $28 \mathrm{~cm}$ in the poloidal direction and the distance between the blades is $12 \mathrm{~cm}$. The distance of the probe along the magnetic field to the nearest limiter blade is about $3 \mathrm{~m}$.

\section{EXPERIMENTAL RESULTS}

Figure 4 displays the measured Mach number in the edge plasma as a function of radius. The plasma flow reverses its direction near the radius of the edge of the ALT-II limiter. In the shadow of this limiter the flow is directed along the field lines towards the nearest limiter surface. In front of ALT-II $\left(r<r_{\mathrm{ALT}-\mathrm{II}}\right)$ the measured Mach number reaches values of up to $M=0.5$. Here the direction of the flow is parallel to the induced plasma current and antiparallel to the toroidal magnetic field direction. For Fig. 5 the conditions for TEXTOR operation are similar to those of Fig. 4, except that the direction of the toroidal magnetic field is inverted. The ion current for $r<r_{\mathrm{ALT} \text {-II }}$ is again in the same direction as the plasma current, i.e. independent of the direction of the toroidal magnetic field. The ion flow direction in the shadow of the ALT-II limiter is, as in Fig. 4, directed towards the nearest ALT-II blade (located - because of the field inversion - in the other direction in the laboratory frame compared with Fig. 4). Therefore, the Mach number profile does not cross the $M=0$ axis in Fig. 5 . For all measurements performed it has been found that the plasma flow in the shadow of the ALT-II limiter is directed towards the nearest limiter surface. For radii $r<r_{\mathrm{ALT} \text {-II }}$ the plasma flow direction is always in the direction of the ohmic plasma current and independent of the direction of the toroidal magnetic field.

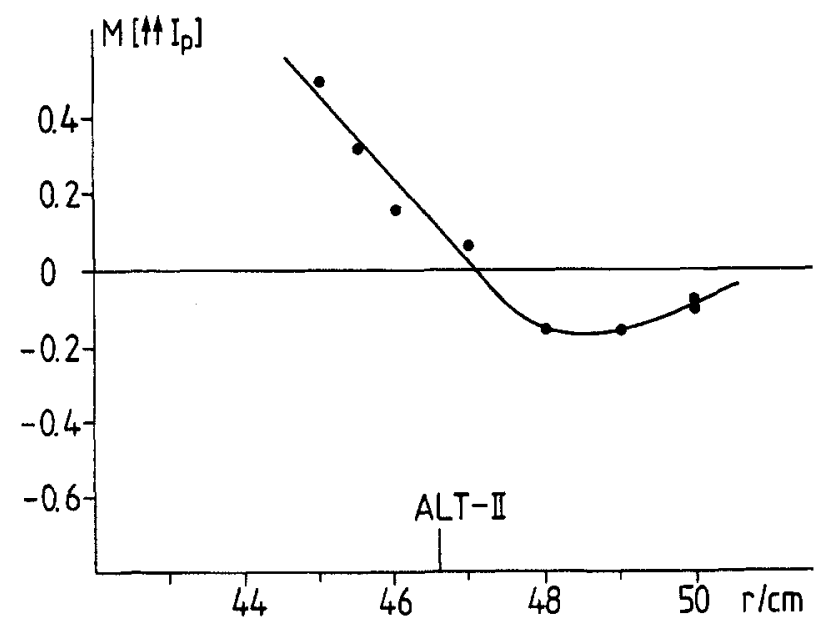

FIG. 4. Radial profile of the Mach number of the plasma flow parallel to the magnetic field at $\mathrm{r}=45 \mathrm{~cm}=\mathrm{r}_{A L T \cdot I I}-1.7 \mathrm{~cm}$, ohmic plasma current and toroidal magnetic field in the opposite direction for $\mathrm{B}_{\text {tor }}=2.25 \mathrm{~T}, \mathrm{I}_{p}=0.35 \mathrm{MA}$ and $\left\langle\mathrm{n}_{e}\right\rangle=2.3 \times 10^{19} \mathrm{~m}^{-3}$.

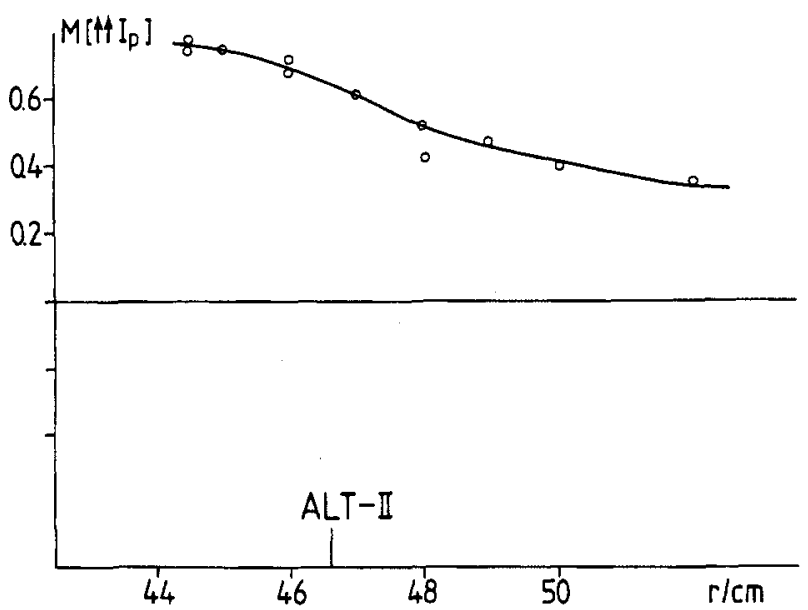

FIG. 5. Radial profile of the Mach number of the plasma flow parallel to the magnetic field at $\mathrm{r}=45 \mathrm{~cm}=\mathrm{r}_{\text {ALT-ll }}-1.7 \mathrm{~cm}$, ohmic plasma current and toroidal magnetic field in the same direction for $\mathrm{B}_{\text {tor }}=2.25 \mathrm{~T}, \mathrm{I}_{p}=0.35 \mathrm{MA}$ and $\left\langle\mathrm{n}_{e}\right\rangle=3.5 \times 10^{19} \mathrm{~m}^{-3}$. 


\section{HÖTHKER et al.}

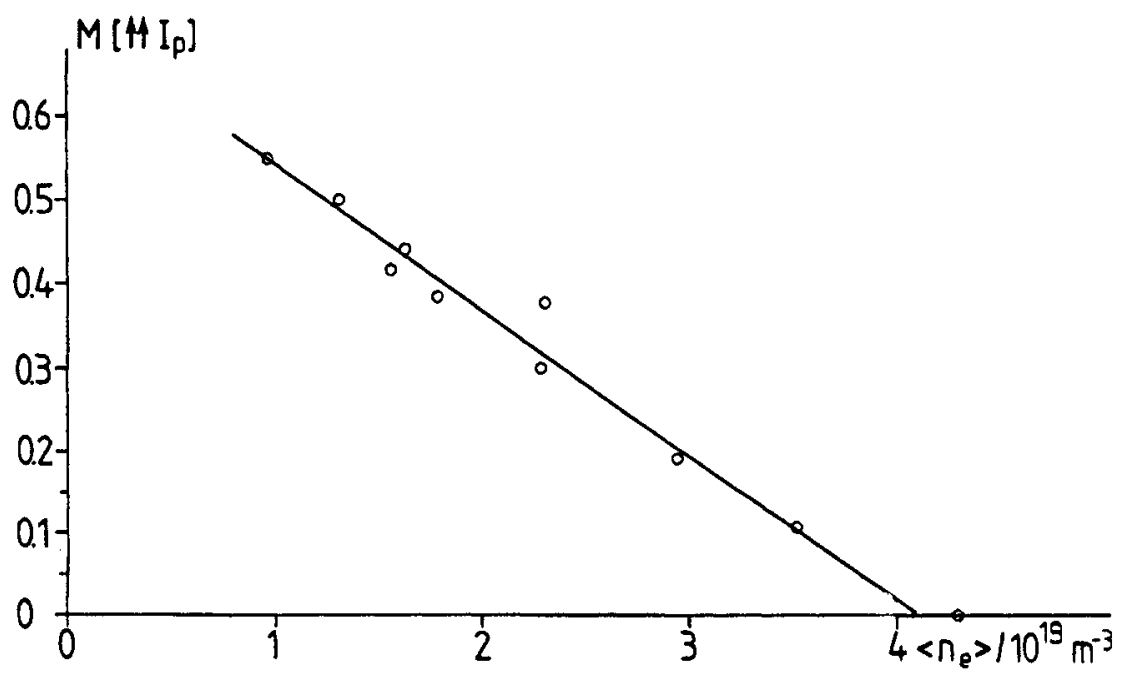

FIG. 6. Mach number of the plasma flow parallel to the magnetic field as a function of the mean core density. Here $\mathrm{r}=\mathrm{r}_{A L T \cdot l l}-1.7 \mathrm{~cm}$, the plasma current and toroidal magnetic field are in opposite directions (standard TEXTOR mode), $\mathrm{B}_{\text {tor }}=2.25 \mathrm{~T}$ and $\mathrm{I}_{p}=0.35$.

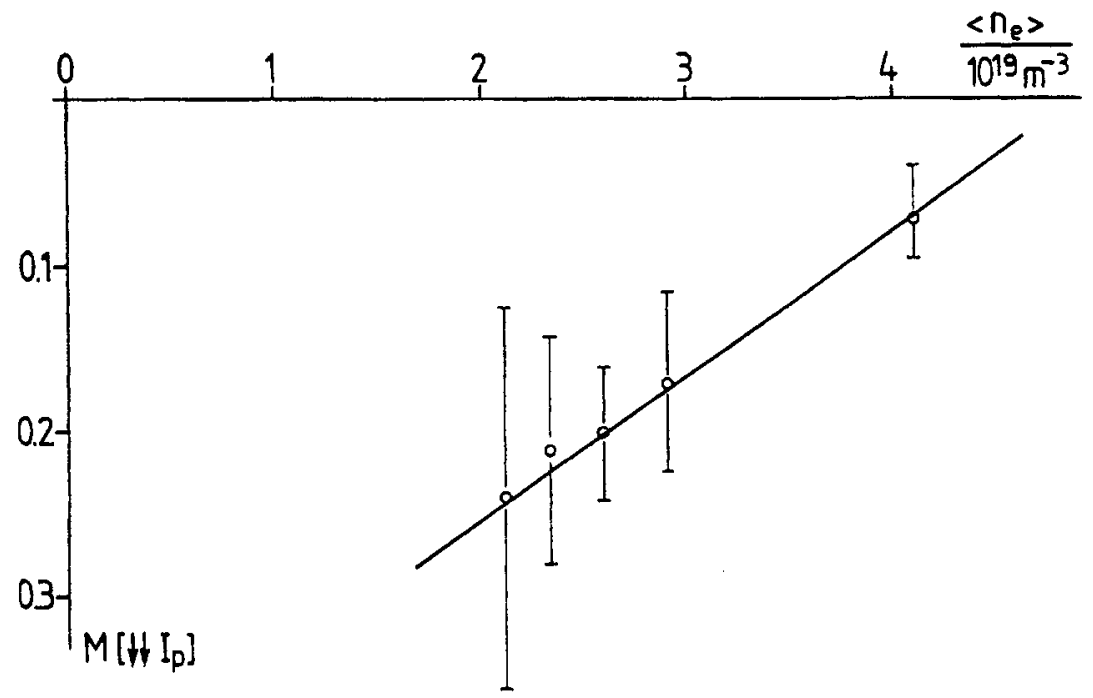

FIG. 7. Mach number of the plasma flow parallel to the magnetic field as a function of the mean core density. Here $\mathrm{r}=\mathrm{r}_{A L T-l}-1.2 \mathrm{~cm}$ and the direction of the plasma current is inverted compared with that in Fig. 6.

The dependence of the Mach number on the mean core density in the shadow of ALT-II and in front of ALT-II is also different: the Mach number in the shadow of ALT-II is almost independent of the core density.

In front of the ALT-II edge, however, the Mach number decreases with increasing mean core density and attains zero, Fig. 6 . For that density $\left\langle n_{\mathrm{e}, \mathrm{cr}}\right\rangle$ the plasma detaches from the limiter. The Mach number scales with the density as

$M /\left(\left\langle n_{\mathrm{e}, \mathrm{cr}}\right\rangle-\left\langle n_{\mathrm{e}}\right\rangle\right)=1.8 \times 10^{20} \mathrm{~cm}^{3}$
A similar result, cf. Fig. 7, has been found for discharges for which the ohmic plasma current is reversed, the direction of the toroidal magnetic field being the same as in Figs 6 to 9. The direction of the plasma flow is again parallel to the direction of the ohmic plasma current and the Mach number of the flow decreases with increasing density and tends to zero when the density approaches the value for detachment.

The profound difference of the plasma conditions in front and in the shadow of the ALT-II limiter is also obvious from the radial profile of the floating potential of 
the probe, cf. Fig. 8 for $\left\langle n_{\mathrm{e}}\right\rangle=4 \times 10^{19} \mathrm{~m}^{-3}$. In the shadow of ALT-II the floating potential is almost independent of the radius while in front of the ALT-II edge it shows a steep gradient.

The results reported up to now are averaged over the stationary phase of the tokamak discharge. In a discharge with exceptionally low noise the Mach number has been determined as a function of time. As with the other investigated discharges it is a normal ohmic discharge in the sense that the energy confinement time $\tau_{E}$ follows the Alcator scaling $\tau_{E} /\left\langle n_{\mathrm{e}}\right\rangle=$ const (Fig. 9). The Mach number measured in front of the ALT-II limiter at the radius $r=r_{\mathrm{ALT}-\mathrm{II}}-1.7 \mathrm{~cm}$ is shown in Fig. 10. During the stationary phase it remains constant as long as the

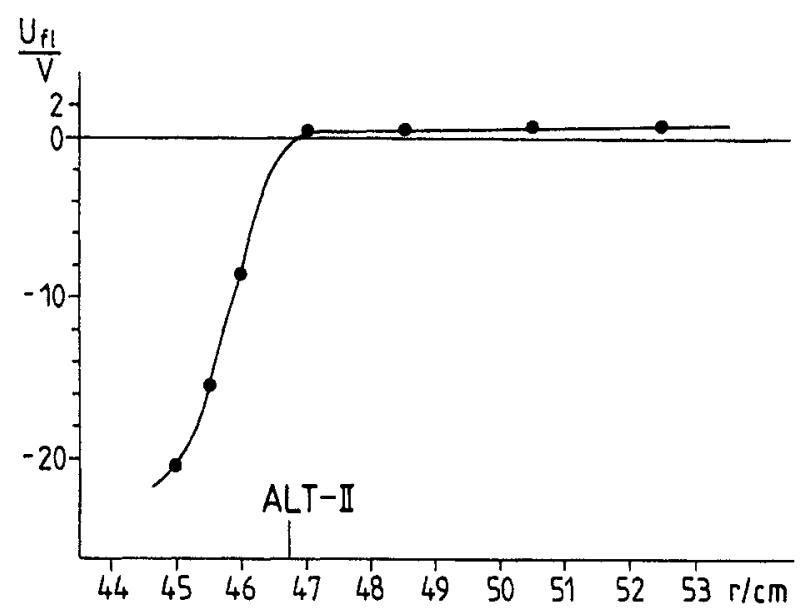

FIG. 8. Electric potential of the floating probe relative to the vessel potential as a function of the radius for $\left\langle\mathrm{n}_{e}\right\rangle=4 \times 10^{19} \mathrm{~m}^{-3}$.

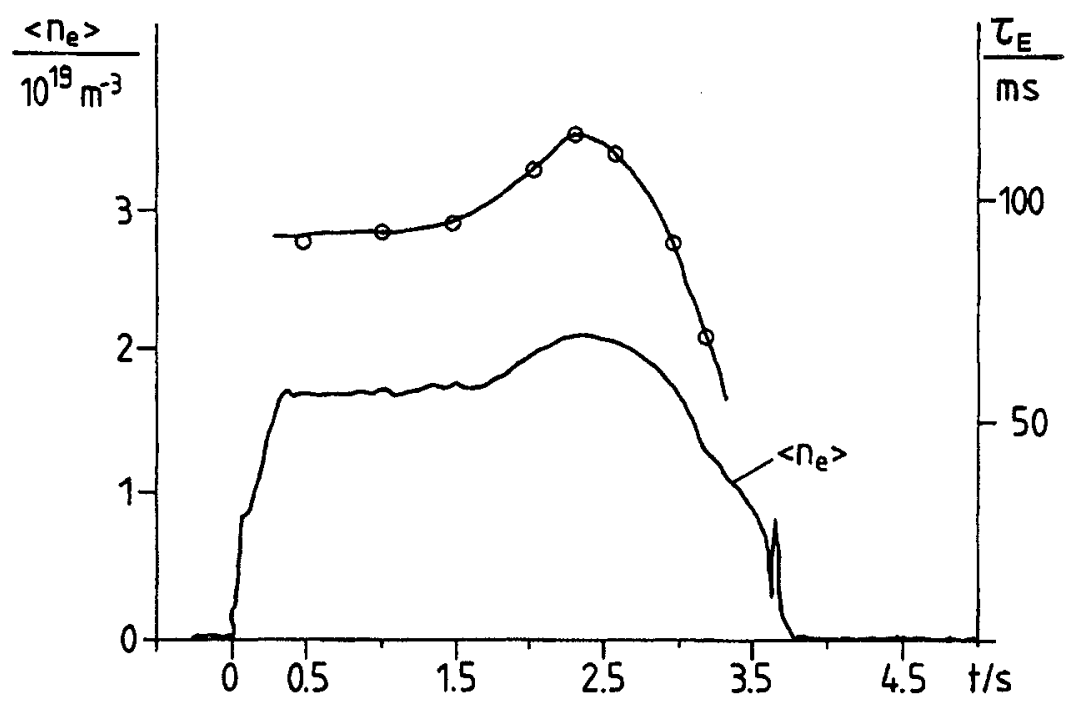

FIG. 9. Mean electron density and energy confinement time as functions of time.

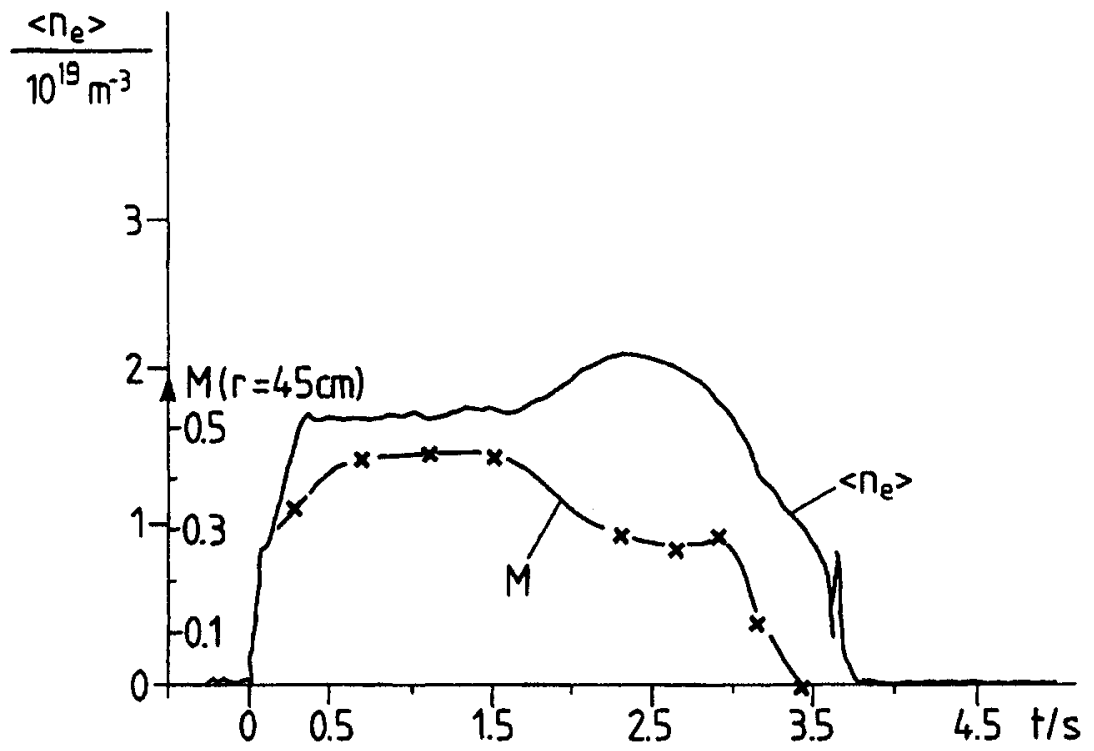

FIG. 10. Mach number and mean core density as functions of time. 


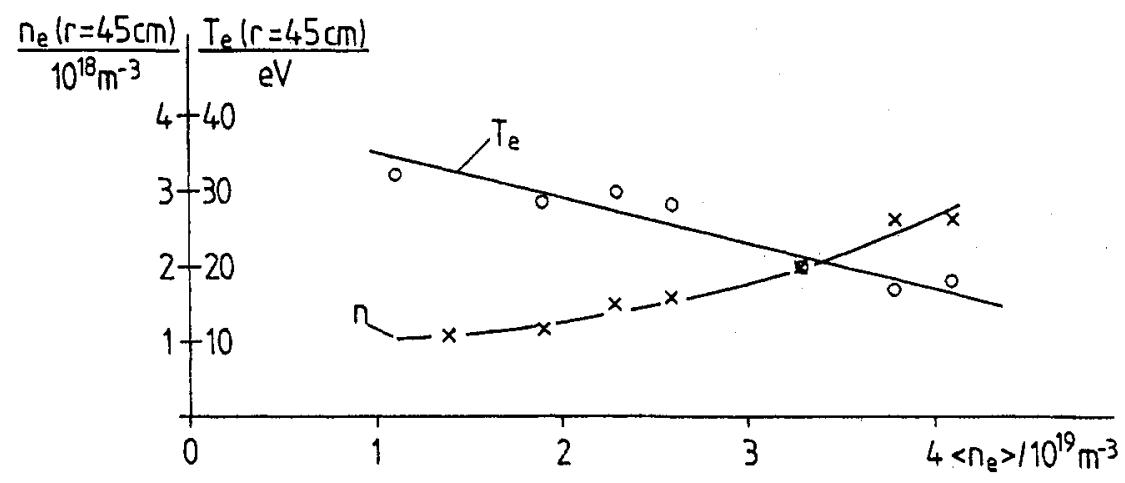

FIG. 11. Electron density and temperature from probe measurements at $\mathrm{r}=45 \mathrm{~cm}$ as functions of the mean core density.

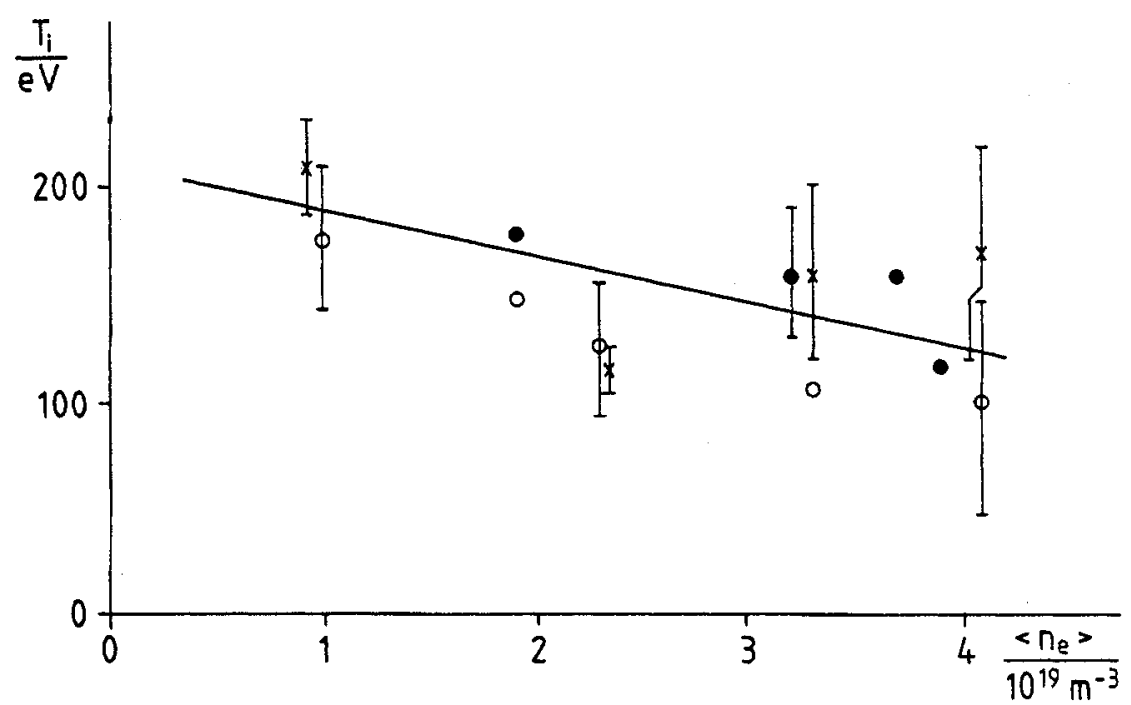

FIG. 12. Ion temperature as a function of the mean core density as determined from the rotating double probe (crosses) at $\mathrm{r}=46 \mathrm{~cm}$ and from lithium beam activated charge exchange spectroscopy at $\mathrm{r}=42 \mathrm{~cm}$ (open circles) and $\mathrm{r}=43 \mathrm{~cm}$ (closed circles) (the latter not measured on the same day).

mean density is constant and decreases when the mean density increases. It follows the same scaling with mean core density as found from Fig. 6 , i.e.

$M /\left(\left\langle n_{\mathrm{e}, \mathrm{cr}}\right\rangle-\left\langle n_{\mathrm{e}}\right\rangle\right)=1.8 \times 10^{-20} \mathrm{~m}^{3}$

The measured flow throughout the discharge again has the same direction as the plasma current.

Figure 11 shows the electron density and temperature at the radial position of the Mach number measurements of Fig. 6 as functions of the mean core density. The density measured by means of the probe and by means of the thermal lithium beam are in fairly good agreement $( \pm 20 \%)$ as we have investigated in more detail already in Ref. [10].
Figure 12 displays the ion temperatures, as determined from the rotating double probe at $r=46 \mathrm{~cm}\left(\mathrm{D}^{+}\right)$and from lithium beam activated charge exchange spectroscopy at $r=42$ and $43 \mathrm{~cm}\left(\mathrm{C}^{6+}\right)[16]$, as functions of the mean core density. The values of the ion temperature from the two methods are the same within the accuracy of the measurements. As the decay length of the ion temperature is relatively large for $r>42 \mathrm{~cm}[16,17]$ $\left(\lambda_{\mathrm{Ti}}>10 \mathrm{~cm}\right.$ for a fit with an exponential function) the comparison of the ion temperatures from the two methods at $r=42 \mathrm{~cm}$ and $r=46 \mathrm{~cm}$ is reasonable.

It is emphasized that the ion temperature is significantly larger than the electron temperature. This result which has been found before by probes in TEXTOR, 
Höthker et al. [5], Amemiya et al. [17] and Matthews et al. [18], is also typical for other tokamaks such as ASDEX, Staib [19], DITE, Stangeby [20] and Matthews et al. [21], and JET, Erents et al. [22] and Weisen et al. [23] - could now also be confirmed by spectroscopic means on TEXTOR $[16,24]$. The mean temperature ratio $T_{\mathrm{i}} / T_{\mathrm{e}}$ at $r=45 \mathrm{~cm}$ in the explored density range is $6.4 \pm 0.7$ and becomes larger owing to the disparity of the ion and electron temperature gradients up to about a value of 10 near the limiter edge, a value which has also been found in JET [18]. (It has been found that the ion temperatures in the edge plasma as measured by the probe and also by the spectroscopic method may differ for the same core plasma conditions by $50 \%$ for series taken at an interval of some weeks. The reason is not yet clear.)

The relatively high ion temperatures are also expected from theory, Hinton and Hazeltine [25], and code calculations, Nicolai and Fuchs [26]. The ion temperatures estimated according to the theory of Ref. [25] are a factor of about two larger than the measured values of Fig. 12.

\section{DISCUSSION OF THE MACH NUMBER MEASUREMENTS}

Each of the measured radial Mach number profiles can be divided into two distinctly different parts separated by the radius $r_{\text {ALT-II }}$ of the edge of the ALT-II limiter. The observations in the shadow of the ALT-II limiter $\left(r>r_{\mathrm{ALT}-\mathrm{II}}\right)$ can be explained by the sink effect of the limiter. This effect has been described in many publications and is not the topic of the present paper.

The essential aim of this paper is to demonstrate that the observations related to the Mach numbers in front of the ALT-II limiter $\left(r<r_{\text {ALT-II }}\right)$ are neoclassical effects. The conditions in the plasma adjacent to the ALT-II edge are such that one has to expect a superposition of the neoclassical toroidal plasma flow and the ion bootstrap current.

First we want to rule out other effects which can also generate a flow which are, however, not important for the present conditions.

(a) It is true that the measurements in front of the ALT-II limiter are performed at radii larger than that of the leading edge of the poloidal limiter

$r_{\text {lim pol }}=r_{\text {ALT-II }}-2.7 \mathrm{~cm}$

This limiter was inserted because it was feared that otherwise the probe would be destroyed in a series of consecutive discharges. The flow characteristics, however, were not modified significantly. This is evident from the following considerations:
- The distance to the poloidal limiter along the magnetic field lines is much larger (60 and $80 \mathrm{~m}$, respectively) than the length of the flux tube of the poloidal limiter $(<10 \mathrm{~m})$. For the case of Fig. 7 the plasma flow is even directed towards the somewhat more distant $(80 \mathrm{~m})$ side of the poloidal limiter.

- The limiters are electrical conductors defining a plane of equal electric potential. In the shadow of ALT-II the influence of this limiter at the position of the probe is evident from the observation that the floating potential of the probe is also constant as a function of the radius, Fig. 8. For radii $r<r_{\text {ALT-II }}$ the floating potential of the probe depends on the radius showing that the poloidal limiter does not dominate the plasma flow in this region.

- It could hardly be understood how a Mach number of, for example, $M=0.5$ could be generated by the sink effect at a position virtually in the plane of symmetry between the two sides of the poloidal limiter.

That the poloidal limiter does not affect the Mach number has been confirmed by one measurement in which it was retracted.

(b) The flow in front of ALT-II $\left(r<r_{\text {ALT-II }}\right)$ is not significantly influenced by the flow in the shadow of ALT-II $\left(r \geq r_{\text {ALT-II }}\right)$, for example, by viscosity. This is evident from the observation that, firstly, the flow direction reverses its sign in Fig. 4 for $r \approx r_{\text {ALT-II }}$ and, secondly, that the Mach number of the flow for $r=$ $r_{\text {ALT-II }}-1.7 \mathrm{~cm}$ remains virtually unchanged when the nearest ALT-II limiter blade is retracted.

(c) The drift speed of the ions due to the induced electric field is much smaller than the observed one.

(d) Since the flow is parallel to the direction of the total magnetic field the flow is not directly generated by the $E \times B$ field.

(e) Since the direction of the observed currents is independent of the direction of the toroidal magnetic field they are not Pfirsch-Schlüter currents.

(f) Since the measurements are performed in the equatorial plane, poloidal asymmetries are not expected to generate the observed flows.

So it is evident that the flows of the plasma and ion bootstrap current extend well into the geometrical shadow of the poloidal limiter owing to the radial diffusion or to the finite width of the banana orbits.

In the following it will be shown that the results of the Mach number measurements are consistent with the related predictions of the neoclassical theory on the toroidal plasma flow and the ion bootstrap current.

Neoclassical theory (see, e.g., Ref. [1]) predicts that the neoclassical plasma flow and the ion bootstrap current 


\section{HÖTHKER et al.}

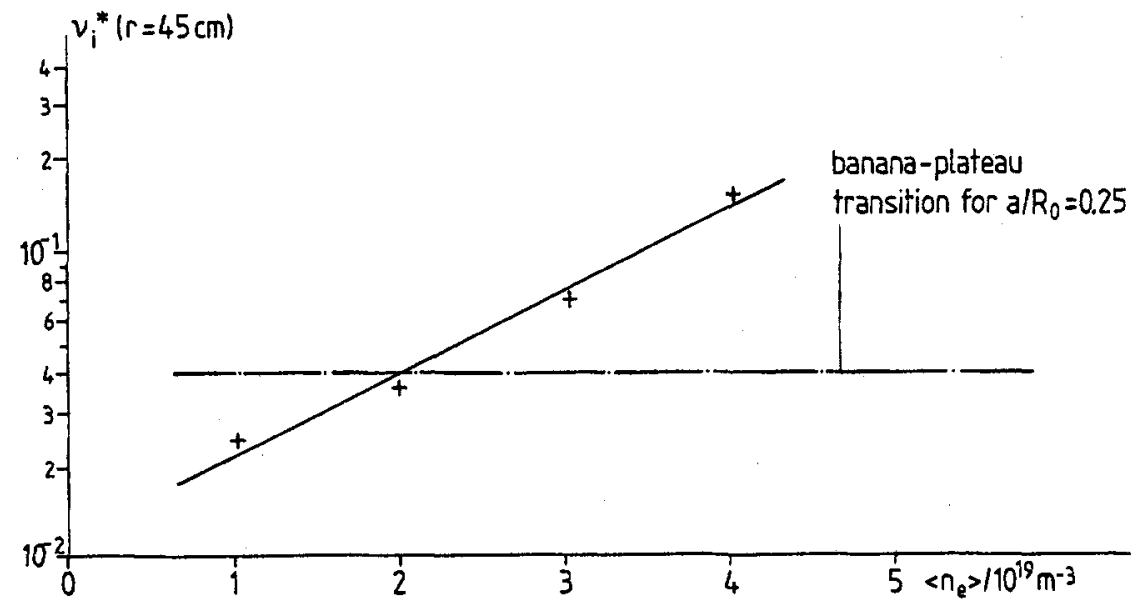

FIG. 13. Ion collisionality at $\mathrm{r}=45 \mathrm{~cm}$ as a function of the mean core density.

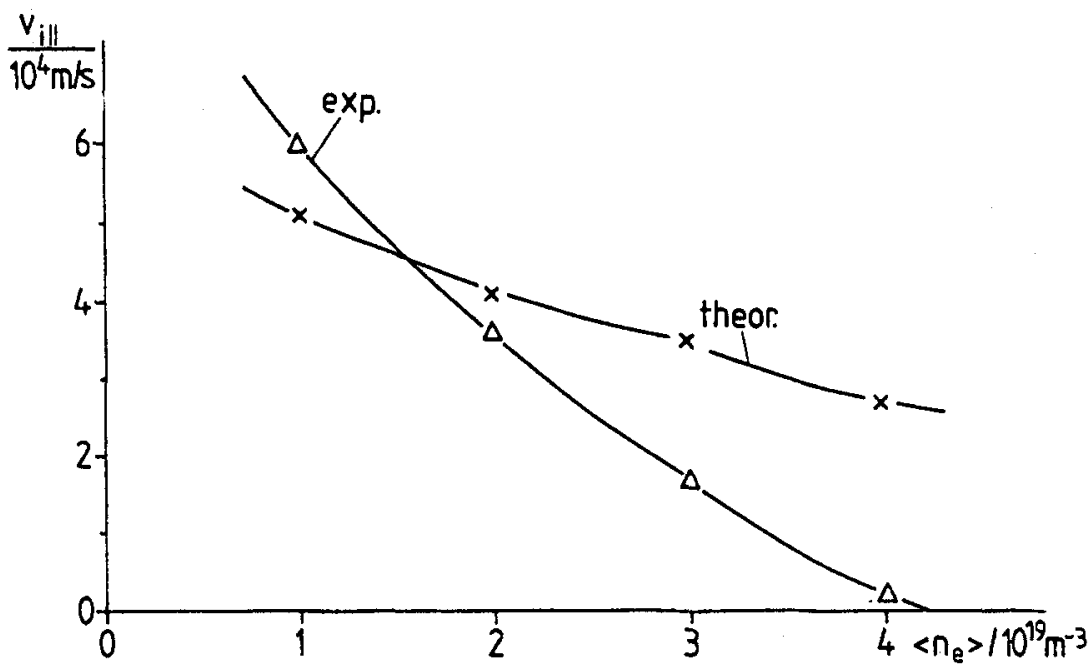

FIG. 14. Comparison of the experimental and theoretical values of the ion speed parallel to the magnetic field at $\mathrm{r}=45 \mathrm{~cm}$ as functions of the mean core density.

have the same directions as the plasma current and are independent of the direction of the toroidal magnetic field. These features are the same as those measured: the direction is observed to be always the same as that of the electrically induced plasma current and it is independent of the direction of the toroidal magnetic field, cf. Figs 6 to 10 ).

Beyond these qualitative agreements suggesting that the measured flow is neoclassical quantitative comparisons also support this interpretation: the neoclassical drift speed of the ions is predicted to be of the order of the thermal ion velocity. For the measured Mach numbers the drift velocity is of the order of the ion sound speed, being of the order of the ion thermal velocity, cf. Figs 6 and 7 .
In Fig. 13 the ion collisionality $\nu_{\mathrm{i}}^{*}$ at $r=r_{\mathrm{ALT}-\mathrm{II}}$ $-1.7 \mathrm{~cm}$ is shown as a function of the mean core density, together with the limit between the banana and plateau regimes for the aspect ratio of the measurements $R_{0} / r \approx 4$ [2]. The discharge conditions are the same as those in Fig. 6. Obviously the ion bootstrap current is expected to contribute to the total ion flow. To our knowledge theoretical results for the plasma conditions of this study have not yet been obtained. Either the results are obtained as being dependent on the aspect ratio but only in the low collisionality limit, Hazeltine et al. [27] and Hirshman [28], or as being dependent on the collisionality but in the large aspect ratio $R / r$ limit, [1]. In the latter case the result for the bootstrap current in the low 
collisionality approximation is

$$
\begin{aligned}
j_{\text {bootstrap }} & =-\frac{(r / R)^{1 / 2} n k T_{\mathrm{e}}}{B_{\theta}}\left[2.44\left(1+\frac{T_{\mathrm{i}}}{Z T_{\mathrm{e}}}\right) \frac{1}{n} \frac{\partial n}{\partial r}\right. \\
& \left.+\frac{0.69}{T_{\mathrm{e}}} \frac{\partial T_{\mathrm{e}}}{\partial r}-\frac{1}{T_{\mathrm{e}}} \frac{\partial \ln T_{\mathrm{i}}}{\partial r}\right]
\end{aligned}
$$

where $B_{\theta}$ is the poloidal magnetic field, $Z$ is the effective ion charge and $k$ is the Boltzmann constant.

However, a quantitative comparison between the experimental and theoretical values of the toroidal plasma flow velocity is possible. For this flow velocity it has been derived [1] that

$v_{\mathrm{i}, \|}=\frac{k T_{\mathrm{i}}}{Z e B_{\theta}}\left[\left(\beta_{1}, g_{2 \mathrm{i}}\right) \frac{\partial \ln T_{\mathrm{i}}}{\partial r}-\frac{\partial \ln p_{\mathrm{i}}}{\partial r}+\frac{Z e}{k T_{\mathrm{i}}} E_{r}\right]$

which at least holds up to the first order in $\sqrt{\epsilon}$, Hazeltine et al. [29] ( $\epsilon$ is the inverse aspect ratio, $\left(\beta_{1}\right.$, $g_{2 \mathrm{i}}$ ) is a coefficient that is slightly dependent on the collisionality).

In Fig. 14 this theoretical velocity is compared with the measured values $v_{\mathrm{i}, \|}=M c_{\mathrm{s}}$ for the conditions of Fig. 6. The density gradient term in the theoretical formula is the dominant one. For mean core densities of about $1 \times 10^{19} \mathrm{~m}^{-3}$ the agreement between the experimental and theoretical values from Eq. (2) is fairly good. But the decrease of the experimental values with increasing density is steeper. This fact is most probably due to the increase of the drag on the ions by charge exchange processes. A theory about neoclassical plasma flow including the effect of neutrals predicts, Valanju et al. [30], that this drag effect on the ions becomes significant for values of the neutral deuterium density of the order of $10^{16} \mathrm{~m}^{-3}$, which is a realistic value for these discharges. The neutral flux is in fact found to increase with increasing core density, Samm et al. [31]. Since the neutral deuterium density has not been measured the determination of the contribution of the ion bootstrap current to the total experimental value from the difference of the two curves in Fig. 14 is difficult. Presuming that $M=1$ is the maximum value of the Mach number an upper limit for the ion bootstrap current density can be inferred from Fig. 14 which is $j_{\text {bootstrap }}=2 \times 10^{4} \mathrm{~A} / \mathrm{m}^{2}$ for the conditions of the discharge with $\left\langle n_{e}\right\rangle=1 \times$ $10^{19} \mathrm{~m}^{-3}$. This is in fair agreement with the theoretical result from Eq. (1), $j_{\text {bootstrap }}=1.5 \times 10^{4} \mathrm{~A} / \mathrm{m}^{2}$. For the experimental aspect ratio $R / r=4$ the low collisionality approximation of Refs [27] and [28] leads to a value of $1 \times 10^{4} \mathrm{~A} / \mathrm{m}^{2}$.
The observations on the speed of the poloidal rotation (if assumed equal to the measured phase velocity) show also, Ivanov et al. [32], that the edge plasma is in the long mean free path neoclassical regime: the neoclassical toroidal plasma flow, being a consequence of the angular momentum balance, is related to the poloidal plasma rotation. The projection of the observed flow parallel to the magnetic field on the poloidal plane has the same direction as the observed poloidal rotation and $v_{\theta} \approx$ $\left(B_{\theta} / B_{\text {tor }}\right) v_{\|}$. The damping of the poloidal neoclassical rotation by ion viscosity has been studied theoretically, Rosenbluth [33]. For binary ion-ion collisions this damping is expected to be insignificant for ion temperatures $T_{\mathrm{i}}>100 Z^{1.6} \mathrm{eV}$, consistent with the measured ion temperatures $(Z$ is the effective ion charge). This relation depends only weakly on the density $\left(\sim n^{2 / 5}\right)$ and holds as quoted for all densities of Fig. 6.

With increasing mean core density the poloidal rotation speed tends to zero for the same density value [32] as the toroidal speed does, Figs 6 and 7.

\section{LIMITATIONS OF THE MEASUREMENTS AND OF THE COMPARISON WITH THEORY}

The essential part of the conclusions relies on the measurement of the Mach numbers. These values are lower limit values because the mean ion Larmor radius is not very small compared with the probe pin radius.

Limited knowledge of the density of the impurities implies an uncertainty of the collisionality. Assuming as an upper limit an impurity level of $10 \%$ and an effective ion charge $Z=3$ in the edge plasma the collisionality would be larger by a factor of about 2 , i.e. the above conclusions would in essence be the same.

As far as the comparison with theory is concerned it should be noted that the locally measured values are compared with the theoretical results of Eq. (2) which are flux averaged values. This may imply a difference of the order of $\epsilon$ or about $25 \%$. Equation (2) has been derived for the weak coupling condition $F-1 \ll 1$ which applies for a plasma with electrons in the Pfirsch-Schlüter regime and ions in the banana regime [1]:

$F-1=\frac{Z(r / R)^{-3 / 2} \sqrt{\frac{m_{\mathrm{e}}}{m_{\mathrm{i}}}} \sqrt{\frac{T_{\mathrm{e}}}{T_{\mathrm{i}}}}}{\nu_{\mathrm{e}}^{*} \nu_{\mathrm{i}}^{*}} \ll 1$

The value of $F-1$ for the density range investigated in Fig. 6 varies over two orders of magnitude, Fig. 15. The weak coupling condition is not satisfied for the entire range of the density. 
HÖTHKER et al.

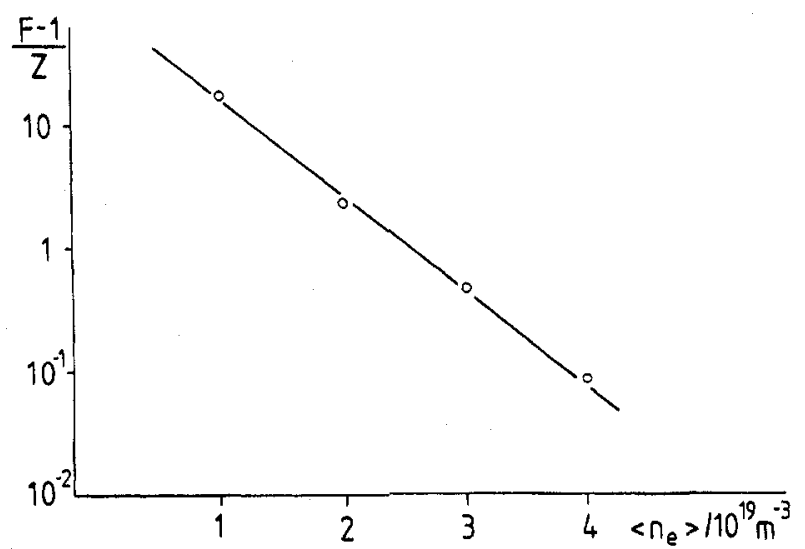

FIG. 15. Weak coupling condition as a function of the mean core density for the plasma parameters of Fig. 6.

Quantitative theoretical results for the bootstrap current density are not available for either the experimental aspect ratio or the collisionality range of this study. The comparison for the low collisionality conditions, however, shows an agreement between the experimental and theoretical results within a factor of 2 .

Furthermore the effect of microturbulence is not incorporated in the theories [1], [27] and [28] that have been used for comparison. This subject has been analysed theoretically by Yushmanov [34, 35], Pereverzev and Yushmanov [36], Connor and Taylor [37], Thyagaraja and Haas [38], Yushmanov [39] and Haas and Thyagaraja [40]. It has been predicted [37, 40], that the enhancement of the bootstrap current due to microturbulence is at most a factor of $(R / r)^{1 / 2}$. Considering that the Mach number decreases, Figs 6 and 7, and that the degree of turbulence increases with increasing density [32] we conclude that for the present conditions the effect of microturbulence is not discernible and probably masked by the effect of charge exchange processes.

\section{APPLICATION OF THE EDGE MODEL ON RESULTS FROM THE ALT-I AND ALT-II PUMP LIMITER OPERATION}

The results from Sections 3 to 5 can elucidate some of the observations on the pump limiters ALT-I and ALT-II, Dippel et al. [41], Finken et al. [42, 43] and Goebel et al. [44].

ALT-I - operational on TEXTOR until 1987 - was mounted in the equatorial plane and the different limiter heads had a limited extension in the poloidal and toroidal directions of typically $30 \times 30 \mathrm{~cm}^{2}$. The particle flow collected by 'scoops' from the ion and electron drift directions were measured separately. The radial distance between the magnetic flux surface tangent to the limiter and the edge of the entrance of the scoops was between $\Delta=1$ and $2 \mathrm{~cm}$. The particle flows into the ALT-I scoops are larger by a factor of 2 to 3 from the ion side than from the electron side, and the flux from the ion side is the larger one also when the plasma current is reversed $[41,42]$. Since the mean banana length is smaller than the connection length of ALT-I and the radial distance of the scoops from the leading edge of ALT-I is of the order of the ion banana width, the collection occurs from a neoclassical plasma. From the considerations in Section 4 one expects therefore the observations on the direction and on the absolute values of the flux asymmetry made on ALT-I.

ALT-II is quasi-symmetric in the toroidal direction and is mounted $45^{\circ}$ below the equatorial plane, as described in Section 2. The geometry of the scoops is similar to that of ALT-I. The connection length of ALT-II is not large compared with the mean banana length. In fact, the discussion of the experimental flow characteristics due to the Mach probe measurements in the shadow of ALT-II, Section 4, gave the result that the neoclassical flow does not extend significantly into the region $r>r_{\text {ALT-II }}$. The flow velocity at the entrance of the scoops is therefore expected to be about the same on the ion and electron sides. But the measured fluxes from the ion and electron sides into the scoops are different $[43,44]$. This difference is attributed, however, to the different densities on the two sides [45, 46]. But the resulting difference of the flow velocity from the ion and electron side into the ALT-II scoops is insignificant. The difference in the density can be explained by the fact that ALT-II is not mounted in the equatorial plane - in contrast to ALT-I - and thus the symmetry of the flux tubes in the scrape-off layer is broken.

Observations on the front face of ALT-II, however, have signatures attributed to a neoclassical plasma as expected: the heat flux is larger on the ion side than that on the electron side for both directions of the plasma current and independent of the direction of the toroidal magnetic field. The asymmetry factor of the heat flow has the value 2 for $\left\langle n_{\mathrm{e}}\right\rangle=1 \times 10^{19} \mathrm{~m}^{-3}$ and approaches unity for increasing density $\left\langle n_{\mathrm{e}}\right\rangle[43]$.

\section{CONCLUSIONS}

The ions in the edge plasma in front of the ALT-II limiter are in the banana/plateau regime. The qualitative flow characteristics expected from neoclassical theory for such plasmas could be confirmed experimentally to a 
large extent. Quantitatively good agreement for small densities has been found. At higher densities there is an in principle agreement with a recent neoclassical theory including the effect of neutrals. For further progress in the quantitative understanding of the edge plasma and especially of its transport properties, measurements of the neutral particle density is imperative. As far as theory is concerned the extension of the theory on bootstrap currents to finite aspect ratios as a function of collisionality is needed as well as the extension to non-weakly-coupled plasmas.

In general, it is compulsory to take the neoclassical character of the ions in the edge plasma into account to understand the particle transport in the edge plasma; but also the energy transport can only be understood if the neoclassical flows having large Mach numbers are included in the analysis.

In particular, the application of the physical picture of the edge plasma developed in this paper allows an understanding of the essential characteristics of particle exhaust by the pump limiters ALT-I and ALT-II and of the energy load on these limiters. The implications of the neoclassical character of the ions in the edge plasma are important and need further investigations as far as the understanding and steering of particle exhaust by pump limiters (and divertors) is concerned.

Finally it may be presumed that further study will lead to a better understanding and control of those core plasma modes that depend critically on edge plasma conditions.

\section{ACKNOWLEDGEMENTS}

It is a great pleasure to thank R. Bengtson, H.A. Claassen, K.H. Finken, H. Gerhauser, R.D. Hazeltine, F.L. Hinton, K. Lackner, A. Nedospasov, J. Neuhauser, A. Nicolai, A. Rogister, E.R. Solano, P.M. Valanju and R. White for constructive discussions and encouragement, and A. Cosler and W. Wirtz for competent technical assistance. Without the excellent performance of TEXTOR and the substantial help of M. Korten in the data acquisition this study would not have been possible.

\section{REFERENCES}

[1] Hinton, F.L., HAZELTine, R.D., Rev. Mod. Phys. 48 (1976) 239

[2] BALESCU, R., Transport Processes in Plasmas, Vol. 2, North-Holland, Amsterdam (1988),

[3] BURRELL, K.H., et al., Phys. Fluids B 2 (1990) 1405.
[4] ZARNSDORFF, M.C., et al., Phys. Rev. Lett. 60 (1988) 1306.

[5] KIM, Y.B., et al., Neoclassical Current and Transport in Auxiliary Heated Tokamaks, Rep. JET-R(88)02, JET Joint Undertaking, Abingdon, Oxfordshire (1988).

[6] BELL, M.G., Nucl. Fusion 19 (1979) 33.

[7] BUGARYA, V.I., et al., JETP Lett. 38 (1983) 405.

[8] HAZELTINE, R.D., Phys. Fluids 17 (1974) 961.

[9] KIM, Y.B., et al., Phys. Fluids B 3 (1991) 2050.

[10] HÖTHKER, K., et al., Rev. Sci. Instrum. 58 (1987) 1046.

[11] HÖTHKER, K, et al., in Controlled Fusion and Plasma Physics (Proc. 14th Eur. Conf. Madrid, 1987), Vol. 11D, Part III, European Physical Society, Geneva (1987) 1272.

[12] HÖTHKER, K., et al., Rev. Sci. Instrum. 61 (1990) 114.

[13] HÖTHKER, K., et al., in Controlled Fusion and Plasma Heating (Proc. 17th Eur. Conf. Amsterdam, 1990), Vol. 14B, Part IV, European Physical Society, Geneva (1990) 1568.

[14] CHUNG, K.S., HUTCHINSON, I.H., Phys. Rev. A 38 (1988) 4721.

[15] HÖTHKER, K., et al., in Controlled Fusion and Plasma Physics (Proc. 16th Eur. Conf. Venice, 1989) Vol. 13B, Part IV, European Physical Society, Geneva (1989) 1525.

[16] SCHORN, R.P., et al., Nucl. Fusion 32 (1992) 351.

[17] AMEMIYA, $\mathrm{H}$., et al., in Asia-Pacific Conf. on Plasma Science and Technology/3rd China-Japan Symp. on Plasma Chemistry/6th China Conf. on Plasma Science and Technology, Nanjing, 1992, Vol. 2, Southeast University, Nanjing (1992) 392.

[18] MATTHEWS, G.F., et al., J. Nucl. Mater. 196-198 (1992) 253.

[19] STAIB, P., J. Nucl. Mater. 111\&112 (1982) 109.

[20] STANGEBY, P.C., Phys. Fluids 28 (1985) 644.

[21] MATTHEWS, G.F., et al., Nucl. Fusion 31 (1991) 1495.

[22] ERENTS, S.K., et al., in Controlled Fusion and Plasma Heating (Proc. 17th Eur. Conf. Amsterdam, 1990), Vol. 14B, Part III, European Physical Society, Geneva (1990) 1385.

[23] WEISEN, H., et al., Nucl. Fusion 31 (1991) 2247.

[24] HEY, J.D., et al., in Controlled Fusion and Plasma Physics (Proc. 20th Eur. Conf. Lisbon, 1993), Vol. 17C, Part III, European Physical Society, Geneva (1993) 1111.

[25] HINTON, F.L., HAZELTINE, R.D., Phys. Fluids 17 (1974) 2236.

[26] NICOLAI, A., FUCHS, G., J. Nucl. Mater. $76 \& 77$ (1978) 556.

[27] HAZELTINE, R.D., et al, Phys. Fluids 16 (1973) 1645.

[28] HIRSHMAN, S.P., Phys. Fluids 31 (1988) 3150.

[29] HAZELTINE, R.D., et al., Phys. Fluids 16 (1973) 1645.

[30] VALANJU, P.M., et al., Phys. Fluids B 4 (1992) 2675.

[31] SAMM, U., et al., Plasma Phys. Control. Fusion 29 (1987) 1321.

[32] IVANOV, R.S., et al., Investigation of $E \times B$ Transport with a Multi Electrode Probe in the Plasma Boundary of TEXTOR, Rep. Jül-2432, Forschungszentrum Jülich $\mathrm{GmbH}$, Jülich (1991).

[33] ROSENBLUTH, M.N., Bull. Am. Phys. Soc. 18 (1973) 1337.

[34] YUSHMANOV, P.N., Sov. Phys.-JETP Lett. 31 (1981) 81.

[35] YUSHMANOV, P.N., Comments Plasma Phys. Control. Fusion 14 (1992) 313.

[36] PEREVERZEV, G.V., YUSHMANOV, P.N., Sov. J. Plasma Phys. 6 (1980) 543. 


\section{HÖTHKER et al.}

[37] CONNOR, J.W., TAYLOR, J.B., Comments Plasma Phys. Control. Fusion 11 (1987) 37

[38] THYAGARAJA, A., HAAS, F.A., Europhys, Lett. 5 (1988) 425.

[39] YUShManOV, P.N., Comments Plasma Phys. Control. Fusion 14 (1988) 313.

[40] HaAS, F.A., THYagarajA, A., Plasma Phys. Control. Fusion 35 (1993) 779.
[41] DIPPEL, K.H., et al., J. Nucl. Mater. 145-147 (1987) 3.

[42] FINKEN, K.H., et al., J. Nucl. Mater. 145-147 (1987) 825

[43] FINKEN, K.H., et al., J. Nucl. Mater. 162-164 (1989) 566.

[44] GOEBEL, D.M., et al., J. Nucl. Mater. 162-164 (1989) 115

[45] SAMM, U., et al., Nucl. Fusion 31 (1991) 1386.

[46] GeRHAUSER, H., ClAASSEN, H.A., J. Nucl. Mater. $176 \& 177$ (1990) 721.

(Manuscript received 15 April 1994

Final manuscript received 21 June 1994) 